Vol. 15, Núm. 4

Oct.-Dic. 2019. pp 239-241 doi: $10.35366 / 94050$

https://dx.doi.org/10.35366/94050

\title{
Recomendaciones de tratamiento de las fracturas del fémur proximal
}

\author{
Treatment recommendations for proximal femoral fractures
}

José Manuel Pérez-Atanasio*

\footnotetext{
* Médico Ortopedista, Adiestramiento en reemplazo articular UNAM, Maestro en Ciencias. Coordinador de Investigación del Hospital Shriners para Niños. Ciudad de México.
}

Correspondencia: José Manuel Pérez-Atanasio. Calle Tanka No. 153 Fraccionamiento Grand Juriquilla, 76226, Querétaro, Querétaro, México. Correo electrónico: drmanuelperezata@gmail.com

\section{RESUMEN}

Con base en las guías de manejo, se tienen las siguientes recomendaciones para tratar las fracturas del fémur proximal: las fracturas transtrocantéricas y subtrocantéricas deben ser tratadas con osteosíntesis. En las fracturas de cuello femoral se debe considerar el desplazamiento y la capacidad de remodelación para una osteosíntesis. No se recomienda el uso de tracción preoperatoria. El operar al paciente dentro de las primeras 48 horas después de la fractura se asocia con mejores resultados. No hay diferencia entre usar una prótesis unipolar o bipolar. La hemiartroplastia es buena opción en pacientes con poca demanda funcional y comorbilidades médicas. En pacientes ancianos se recomienda el uso de vástagos cementados. Parece existir un mayor riesgo de luxación cuando se utiliza el abordaje posterior.

Palabras clave: Fracturas de fémur proximal, guía de recomendación, artroplastia.

\section{ABSTRACT}

Based on the guidelines, the following recommendations are available for treating fractures of the proximal femur: Transtrocatheric and sub-trochanteric fractures should be treated with osteosynthesis. In femoral neck fractures, displacement and remodeling capacity for osteosynthesis should be considered. The use of preoperative traction is not recommended. Operating the patient within the first 48 hours after the fracture is associated with better results. There is no difference between wearing a unipolar or bipolar prosthesis. Hemi arthroplasty is a good option in patients with little functional demand and medical comorbidities. In elderly patients, the use of cemented stems is recommended. There appears to be an increased risk of dislocation when the posterior approach is used.

Keywords: Proximal femoral fractures, guidelines, arthroplasty.

El objetivo de este documento es definir las recomendaciones para el tratamiento de las fracturas del fémur proximal, por lo que debemos empezar diciendo que el fémur proximal se divide en las siguientes regiones: cabeza femoral, región intracapsular que corresponde al cuello femoral, región transtrocantérica y subtrocantérica, esta división se muestra en la Figura 1. 
Para la elaboración de este artículo se consultaron los siguientes documentos:

- Guía de práctica clínica: Manejo médico integral de fractura de cadera en el adulto mayor. Instituto Mexicano del Seguro Social. ${ }^{1}$

- Guía de práctica clínica: Diagnóstico y tratamiento de fracturas intracapsulares del extremo proximal del fémur de la Secretaría de Salud. ${ }^{2}$

- Guía de práctica clínica para el tratamiento de la fractura de cadera en el adulto mayor de la Academia Americana de Cirujanos Ortopédicos (AAOS). ${ }^{3}$

- Guía para el tratamiento de la fractura de cadera del Instituto Nacional de Salud y Excelencia clínica del Reino Unido. ${ }^{4}$

- Guía de Australia y Nueva Zelanda para el tratamiento de la fractura de cadera. ${ }^{5}$

\section{RECOMENDACIONES}

Las fracturas que se presenten en la región transtrocantérica o subtrocantérica deben ser tratadas con osteosíntesis, evitando el tratamiento con artroplastia.

El tratamiento de artroplastia se recomienda para fracturas intracapsulares y de cabeza femoral. Con respecto a las fracturas de cabeza femoral, en prácticamente todos los casos se recomienda utilizar artroplastia.

El debate existe en las fracturas de cuello o de la región intracapsular, en términos generales se recomienda la osteosíntesis para jóvenes con potencial de remodelación, así como en las fracturas estables no desplazadas, debiendo favorecer las técnicas no invasivas, como la colocación de tornillos canulados con ayuda de fluoroscopio. Para las fracturas de cuello femoral desplazadas e inestables, existe evidencia sólida para recomendar que sean tratadas con artroplastia.

Tracción preoperatoria. No se recomienda el uso de tracción preoperatoria.

Tiempo de espera para la cirugía. El operar al paciente dentro de las primeras 48 horas posterior a la fractura se asocia con mejores resultados, como se demuestra en la literatura científica.

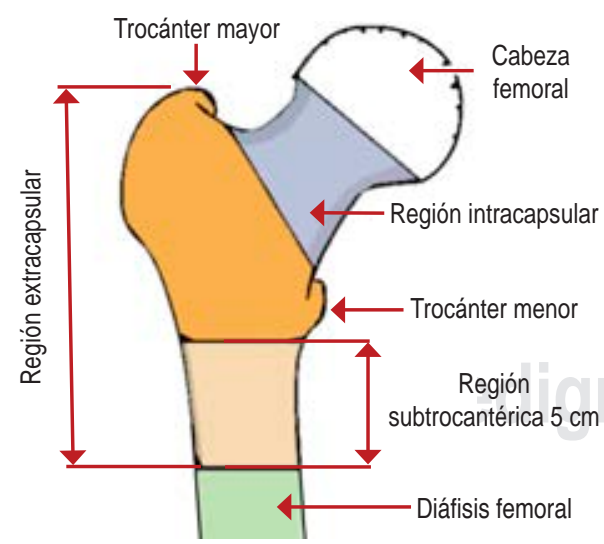

Figura 1: Regiones del fémur proximal.

Prótesis unipolar vs bipolar. Los resultados clínicos postoperatorios son similares con ambos tipos de prótesis.

Hemi vs artroplastia total de cadera. Moderada evidencia científica avala la recomendación de preferir la artroplastia total de cadera; sin embargo, la hemiartroplastia parece ser una excelente opción en pacientes con poca demanda funcional y comorbilidades médicas. Otro elemento a considerar es la posibilidad de luxación de una ATC por la debilidad de los músculos, principalmente del glúteo 
medio en pacientes ancianos. La hemiprótesis es estable mecánicamente y tiene bajo riesgo de luxación.

Uso de vástagos cementados. En pacientes ancianos se recomienda el uso de vástagos cementados, con moderada evidencia científica que soporte esta recomendación.

Abordaje quirúrgico. Parece existir un mayor riesgo de luxación cuando se utiliza el abordaje posterior en pacientes con fracturas de cuello femoral; sin embargo, no existe una evidencia científica sólida que soporte esta aseveración, por lo que la experiencia del cirujano sigue siendo un factor determinante para la elección del abordaje.

\section{BIBLIOGRAFÍA}

1. Manejo Médico Integral de Fractura de Cadera en el Adulto Mayor. México: Instituto Mexicano del Seguro Social; 08/07/2014.

2. Diagnóstico y tratamiento de fracturas intracapsulares del extremo proximal del fémur. México: Secretaría de Salud; 2009.

3. Management of hip fractures in the elderly: evidence-based clinical practice guideline. Adopted by the American Academy of Orthopaedic Surgeons (AAOS) Board of Directors September 5, 2014.

4. National Institute for Health and Clinical Excellence. Evidence Update 34-Hip fracture. London: National Institute for Health and Clinical Excellence (NICE). [Last updated 10 May 2017] Available in: https:// www.nice.org.uk/terms-and-conditions\#notice-ofrights

5. Australian and New Zealand Hip Fracture Registry (ANZHFR) Steering Group. Australian and New Zealand Guideline for Hip Fracture Care: Improving Outcomes in Hip Fracture Management of Adults. Sydney: Australian and New Zealand Hip Fracture Registry Steering Group; 2014. 